\title{
Adsorption of Microstructured Particles at Liquid-Liquid Interfaces
}

\author{
Yoshimune Nonomura, ${ }^{*}$, Shigeyuki Komura, ${ }^{\ddagger}$ and Kaoru Tsujii ${ }^{\S}$ \\ Tokyo Research Laboratories, Kao Corporation, 2-1-3 Bunka, Sumida-ku, Tokyo 131-8501, Japan, \\ Department of Chemistry, Faculty of Science, Tokyo Metropolitan University, 1-1 Minami-Osawa, Hachioji-shi, \\ Tokyo 192-0397, Japan, and Nanotechnology Research Center, Research Institute for Electronic Science, \\ Hokkaido University, N21, W10 Kita-ku, Sapporo 001-0021, Japan
}

Received: March 19, 2006; In Final Form: May 9, 2006

The solid particles are adsorbed at interfaces and form self-assembled structures when the particles have suitable wettability to both liquids. Here, we show theoretically how the microstructure on the particle surface affects their adsorption properties. The physical properties of the interface adsorbing a particle will be described by taking into account the surface roughness due to the microstructure. The microstructure on the surface changes drastically the wettability and the equilibrium position of the adsorbed particle. Therefore, the contact angle of the particle at the three-phase contact line shifts with the particle surface area, because the surface roughness enhances the interfacial properties of the particle surface. Moreover, the range of the interfacial tensions at which the particle is adsorbed becomes narrower with the increase of the surface roughness. The effect of the particle shape on the adsorption properties is also studied. In the case of disk-shaped particles, the energy changes discontinuously when the plane surface of the particle contacts the liquid-liquid interface. The adsorbing position does not change with the surface roughness. The orientation of a parallelepiped particle at the liquid-liquid interface is governed by the aspect ratio and the surface area of the particle. On the other hand, the particle which is partially covered with the microstructured surface is adsorbed firmly at the interface in an oriented state. We should consider not only the interfacial tensions but also the surface structure and the particle shape to control the adsorption behavior of the particle.

\section{Introduction}

The surface-active adsorbed particles align at liquid-liquid interfaces ${ }^{1,2}$ and stabilize emulsions and foams. ${ }^{3,4}$ These emulsions and foams have been applied to food or cosmetic products, because they are stable due to the adsorption of the particles on the interfaces..$^{5,6}$ In recent years, some scientists have focused on the emulsions as a template of functional composite materials. A hollow sphere and a ribosome-like nanocapsule were obtained through the emulsion state. ${ }^{7-9}$

The mechanism for the adsorption of the surface-active particle has been discussed on the basis of the interfacial energy argument. ${ }^{10,11}$ The equilibrium position of the adsorbed particle can be predicted from the interfacial energy. When spherical particles are adsorbed at an interface, the energy depends on the three different interfacial tensions, that is, between the particle-liquid interfaces and a liquid-liquid interface. The particle is adsorbed at the interface when the interfacial tensions among the three phases are balanced according to Young's equation.

The interfacial properties of the particles with the microstructured surface are interesting due to their great potential as industrial materials, such as electronic materials, medical materials, and supported catalysts. ${ }^{12}$ Moreover, complicated rough structures exist on the surfaces of natural products, such as skins, leaves, and biominerals. These structures on the solid surfaces change their wettability drastically. For example, Tsujii et al. showed that the fractal surface of a wax, alkylketene dimer,

\footnotetext{
$\dagger$ Kao Corporation.

$\doteqdot$ Tokyo Metropolitan University.

$\S$ Hokkaido University.
}

is super-water-repellent. ${ }^{13-16}$ In general, wettability of the microstructured surface has been discussed on the basis of the Wenzel model. ${ }^{17,18}$ The interfacial energy is proportional to the surface area. However, the model does not work when the surface roughness is above a critical value. In such a case, the Cassie-Baxter model is more suitable than the Wenzel model. ${ }^{19}$ In the latter model, it is assumed that the rough surface consists of more than one component.

In the present work, we have calculated the interfacial energy of the liquid-liquid interface adsorbing a spherical particle with a microstructured surface, as illustrated in Figure 1a. We study the effect of the microstructured rough surface on the interfacial energy. The surface area of the particle is assumed to be $r$ times larger than that of the particle having a smooth surface. ${ }^{20}$ The quantity $r$ is designated as the surface area magnification factor. We can obtain $r$ by measuring the specific surface area or by analyzing the surface geometry based on the concept of fractal. ${ }^{13}$ The interfacial energy can be calculated by taking into account the increase of the particle surface area.

The interfacial energy of the liquid-liquid interface adsorbing the disk-shaped particle, the parallelepiped particles, or the spherical particle which are partially covered with the microstructured surface is also derived to discuss the effect of the particle shape. In general, asymmetric particle shape and partial modification of the particle surface cause the particle to be adsorbed with a fixed orientation at the liquid-liquid interface. ${ }^{20-23}$ Some preparation methods for the asymmetric particle have been proposed recently. ${ }^{24,25}$ We demonstrate here that the particle shape and the partial modification of the particle surface are the most effective factors for controlling the adsorbing state of the particle. 
(a) Spherical particle

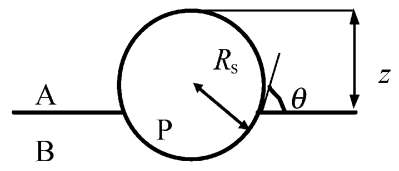

(c) Parallelepiped particle

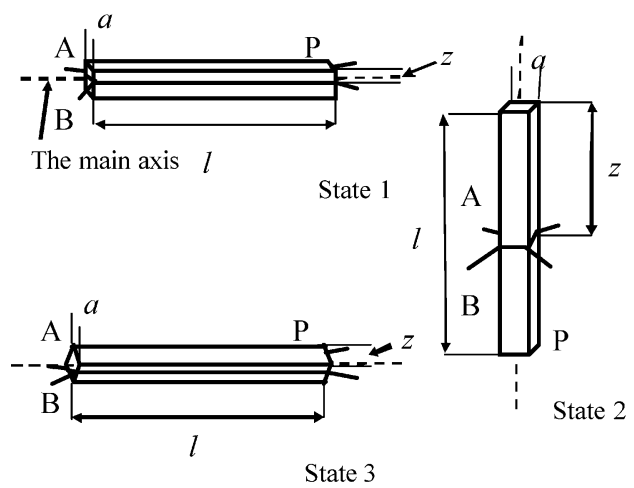

(b) Disk-shaped particle

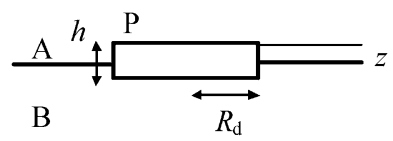

(d) Partially microstructured particle
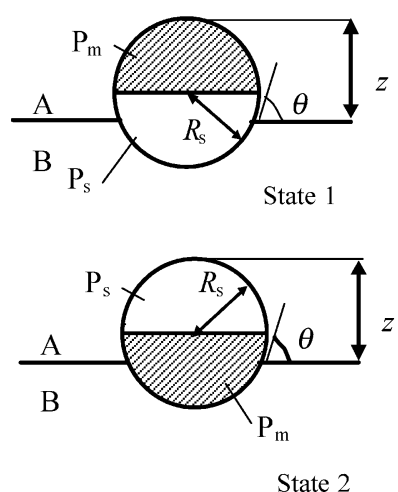

Figure 1. Schematic illustrations of the interface adsorbing (a) a spherical particle, (b) a disk-shaped particle, (c) a parallelepiped particle, and (d) a partially microstructured particle. The particles $\mathrm{P}$ are adsorbed at the interface between liquids $\mathrm{A}$ and $\mathrm{B}$. In the illustrations, $R_{\mathrm{S}}$ and $R_{\mathrm{d}}$ are the radius of the spherical particle and the disk-shaped particle, respectively. The length $h$ is the height of the disk-shaped particle. The quantity $a$ is the length of one side of the square, $l$ is the height of the rectangular parallelepiped particle, and $z$ is the immersion depth of the particle. The angle $\theta$ is the contact angle at the three-phase contact line.

\section{Results}

(a) Energy of the Liquid-Liquid Interface Adsorbing the Spherical Particle with the Microstructured Surface. We now discuss the adsorption of spherical particle $\mathrm{P}$ at the liquidliquid interface, as shown in Figure 1a. Let $F$ be the interfacial energy per particle when it is adsorbed at the interface between liquids $\mathrm{A}$ and $\mathrm{B}$. Then, $F$ is given by $F=4 \pi r R_{\mathrm{s}}{ }^{2} \gamma_{\mathrm{AP}}$ or $F=$ $4 \pi r R_{\mathrm{S}}{ }^{2} \gamma_{\mathrm{BP}}$ when the whole particle is immersed in either liquid A or liquid $\mathrm{B}$, respectively. Here, $R_{\mathrm{S}}$ is the radius of the particle and $\gamma_{i j}$ is the interfacial tension between phases $i$ and $j$. When the particle is adsorbed at the interface between liquids $\mathrm{A}$ and $\mathrm{B}$, as shown in Figure 1a, $F$ is given by eq 1 :

$$
F=4 \pi r R_{\mathrm{s}}^{2} \gamma_{\mathrm{BP}}-2 \pi R_{\mathrm{s}} \gamma_{\mathrm{AB}}(1-r \Sigma) z+\pi \gamma_{\mathrm{AB}} z^{2}
$$

where $\Sigma$ is $\left(\gamma_{\mathrm{AP}}-\gamma_{\mathrm{BP}}\right) / \gamma_{\mathrm{AB}}$ and $z$ is the depth of immersion into liquid $\mathrm{A}$. The symbol $\Sigma$ is equal to minus cosine of the contact angle for the particle with the smooth surface. The derivation of the interfacial energy, $F$, is presented in the Supporting Information. In this interfacial energy, we have taken into account both the contact of the particle surface with liquid $\mathrm{A}$ and the elimination of the interface between liquids $\mathrm{A}$ and B. The surface roughness changes the former factor, and the quantity $r$ is involved in the first and second terms. We plot dimensionless $F /\left(\pi R_{\mathrm{S}}^{2} \gamma_{\mathrm{AB}}\right)$ against $z / R_{\mathrm{S}}$ when $\gamma_{\mathrm{AP}} / \gamma_{\mathrm{AB}}=0.4$, $\gamma_{\mathrm{BP}} / \gamma_{\mathrm{AB}}=0.5$, and $r=3$ in Figure $2 \mathrm{a}$. The above energy $F$ attains a minimum at $z_{\min }$ :

$$
z_{\min }=(1-r \Sigma) R_{\mathrm{s}}
$$

The corresponding minimized energy, $F_{\min }$, is given by

$$
F_{\min }=4 \pi r R_{\mathrm{s}}^{2} \gamma_{\mathrm{BP}}-\pi R_{\mathrm{s}}^{2} \gamma_{\mathrm{AB}}(1-r \Sigma)^{2}
$$

where the first term is the energy when the particle is immersed in liquid B and the second term is the energy change due to the adsorption of the particle at the liquid-liquid interface. Here, we define the energy gaps between $F_{\min }$ and the energy when the particle is immersed in liquid $\mathrm{B}(\mathrm{A})$ as $\Delta F_{1}\left(\Delta F_{2}\right)$ (see Figure $2 a)$. These energy changes are important, because the particle adsorbs at the liquid-liquid interface when $\Delta F_{1}, \Delta F_{2}>0$. For the spherical particle, they are given by $\Delta F_{1}=\pi R_{\mathrm{S}}^{2} \gamma_{\mathrm{AB}}(1-$ $r \Sigma)^{2}$ and $\Delta F_{2}=4 \pi r R_{\mathrm{s}}^{2} \gamma_{\mathrm{AB}} \Sigma+\pi R_{\mathrm{s}}{ }^{2} \gamma_{\mathrm{AB}}(1-r \Sigma)^{2}$, respectively. The energy changes, $\Delta F_{1}$ and $\Delta F_{2}$, depend on the square of $r$, whereas $z_{\min }$ is proportional to $r$. In Figure $2 \mathrm{~b}$, we plot $\Delta F_{1}$, $\Delta F_{2}$, and $z_{\min }$ as a function of $r$ when $\gamma_{\mathrm{AP}} / \gamma_{\mathrm{AB}}=0.4$ and $\gamma_{\mathrm{BP}} /$ $\gamma_{\mathrm{AB}}=0.5$. The energy change $\Delta F_{2}$ decreases with $r$, while $\Delta F_{2}$ and $z_{\min }$ increase. The particle shifts to liquid A with an increase of $r$, and it is adsorbed at the liquid-liquid interface when $r<$ 10.

The state diagram of the spherical particle against $\Sigma$ and $r$ is shown in Figure 2c. The spherical particle adsorbs at the interface when the particle satisfies $-r^{-1}<\Sigma<r^{-1}$. The particle is dispersed in liquid A (B) if $\Sigma$ is smaller than $-r^{-1}$ (larger than $r^{-1}$ ). The above range of the interfacial tensions narrows with the increase of $r$. The allowed range for adsorptiopn is $-1<\Sigma<1$ when $r=1$, whereas it is $-0.2<\Sigma<0.2$ when $r=5$.

(b) Energy of the Interface Adsorbing the Disk-Shaped Particle with the Microstructured Surface. We discuss the adsorption of the disk-shaped particle at the liquid-liquid interface, as shown in Figure 1b. The interfacial energy, $F$, is given by $F=2 \pi r R_{\mathrm{d}}\left(R_{\mathrm{d}}+h\right) \gamma_{\mathrm{AP}}$ or $F=2 \pi r R_{\mathrm{d}}\left(R_{\mathrm{d}}+h\right) \gamma_{\mathrm{BP}}$ when the whole particle is immersed either in liquid $\mathrm{A}$ or in liquid $\mathrm{B}$, respectively. Here, $R_{\mathrm{d}}$ and $h$ are the radius and the height of the particle, respectively. When the particle is adsorbed at the interface between liquids $\mathrm{A}$ and $\mathrm{B}, F$ is given by eq 4 :

$$
F=2 \pi r R_{\mathrm{d}}\left(R_{\mathrm{d}}+h\right) \gamma_{\mathrm{BP}}-\pi R_{\mathrm{d}}^{2} \gamma_{\mathrm{AB}}(1-r \Sigma)+2 \pi r R_{\mathrm{d}} \gamma_{\mathrm{AB}} \Sigma z
$$

The derivation of the interfacial energy, $F$, is presented in the Supporting Information. This interfacial energy changes with $z$, as shown in Figure 3a. We have plotted dimensionless $F /\left(\pi R_{\mathrm{d}}^{2} \gamma_{\mathrm{AB}}\right)$ when $\gamma_{\mathrm{AP}} / \gamma_{\mathrm{AB}}=0.4, \gamma_{\mathrm{BP}} / \gamma_{\mathrm{AB}}=0.5, h / R_{\mathrm{d}}=0.1$, and $r=3$. It is remarkable that the energy changes discontinu- 

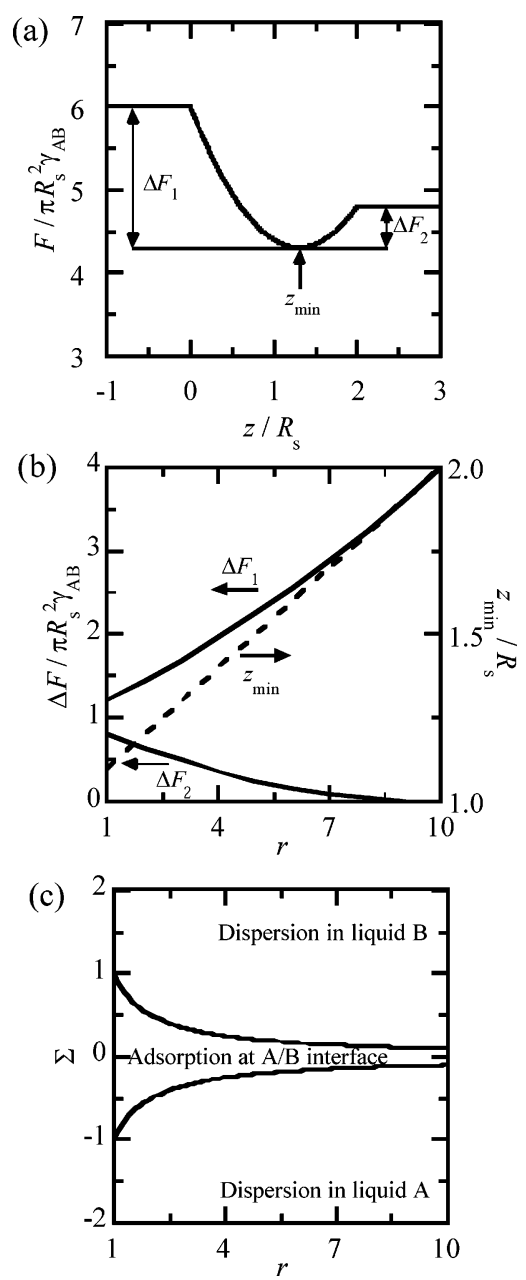

Figure 2. Adsorbing state of the spherical particle. (a) The dimensionless adsorption energy, $F /\left(\pi R_{\mathrm{s}}^{2} \gamma_{\mathrm{AB}}\right)$, against immersion depth, $z / R_{\mathrm{s}}$, for the spherical particle $\mathrm{P}$ at constant radius $R_{\mathrm{S}}$ of the particle and the interfacial tensions $\gamma_{i j}$. The parameters are as follows: $\gamma_{\mathrm{AP}} / \gamma_{\mathrm{AB}}=0.4$, $\gamma_{\mathrm{BP}} / \gamma_{\mathrm{AB}}=0.5$, and $r=3$. (b) The energy gaps, $\Delta F_{1}$ and $\Delta F_{2}$, and the equilibrium position of the particle, $z_{\mathrm{min}}$, against the ratio of the particle surface area, $r$, when $\Sigma=-0.1$. (c) The state diagram of the particle against the interfacial tensions, $\gamma_{i j}$, and the surface area magnification factor, $r$.

ously at $z=0$ and $h$, that is, when the plane surface of the particle contacts the liquid-liquid interface. The energy changes linearly with $z$ as a result of the contact of the side surface with liquid $\mathrm{A}$ and attains the minimum at $z=h$. The corresponding minimized energy is given by

$$
\begin{array}{r}
F_{\text {min }}=2 \pi r R_{\mathrm{d}}\left(R_{\mathrm{d}}+h\right) \gamma_{\mathrm{BP}}-\pi R_{\mathrm{d}}{ }^{2} \gamma_{\mathrm{AB}}(1-r \Sigma)+ \\
2 \pi r R_{\mathrm{d}} h \gamma_{\mathrm{AB}} \Sigma
\end{array}
$$

where the first term is the energy when the particle is immersed in liquid $\mathrm{B}$ and the second and third terms are the energy changes due to the adsorption of the particle at the liquidliquid interface. The energy gaps, $\Delta F_{1}$ and $\Delta F_{2}$, are given by $\Delta F_{1}=\pi R_{\mathrm{d}}^{2} \gamma_{\mathrm{AB}}-\pi r R_{\mathrm{d}}\left(R_{\mathrm{d}}+2 h\right) \gamma_{\mathrm{AB}} \Sigma$ and $\Delta F_{2}=\pi R_{\mathrm{d}}^{2} \gamma_{\mathrm{AB}}+$ $\pi r R_{\mathrm{d}}^{2} \gamma_{\mathrm{AB}} \Sigma$, respectively.

In Figure $3 \mathrm{~b}$, we plot $\Delta F_{1}, \Delta F_{2}$, and $z_{\min }$ as a function of $r$ when $\gamma_{\mathrm{AP}} / \gamma_{\mathrm{AB}}=0.4, \gamma_{\mathrm{BP}} / \gamma_{\mathrm{AB}}=0.5$, and $h / R_{\mathrm{d}}=0.1$. The energy changes, $\Delta F_{1}$ and $\Delta F_{2}$, are proportional to $r$, whereas $z_{\min }$ does not depend on $r$, because the interfacial energy is minimized when the plane surface is in contact with the liquid-liquid interface irrespective of the surface area. The disk-shaped particle adsorbs at the liquid-liquid interface when $r<10$, because $\Delta F_{1}, \Delta F_{2}>0$.
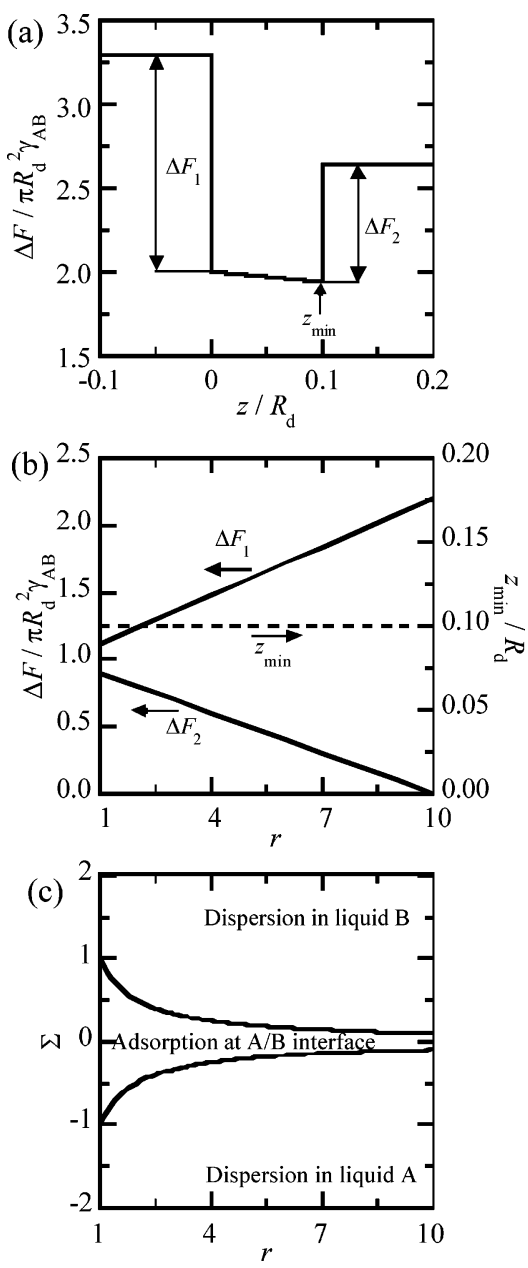

Figure 3. Adsorbing state of the disk-shaped particle. (a) The dimensionless adsorption energy, $F /\left(\pi R_{\mathrm{d}}^{2} \gamma_{\mathrm{AB}}\right)$, against immersion depth, $z / R_{\mathrm{d}}$, for the disk-shaped particle at constant radius $R_{\mathrm{d}}$ of the particle and the interfacial tensions $\gamma_{i j}$. The parameters are as follows: $\gamma_{\mathrm{AP}} /$ $\gamma_{\mathrm{AB}}=0.4, \gamma_{\mathrm{BP}} / \gamma_{\mathrm{AB}}=0.5, h / R_{\mathrm{d}}=0.1$, and $r=3$. (b) The energy gaps, $\Delta F_{1}$ and $\Delta F_{2}$, and the equilibrium position of the particle, $z_{\min }$, against the ratio of the particle surface area, $r$. (c) The state diagram of the particle against the interfacial tensions, $\gamma_{i j}$, and the surface area magnification factor, $r$.

The state diagram for the disk-shaped particle is shown in Figure $3 \mathrm{c}$. The required condition is similar to that for the spherical particle. The disk-shaped particle adsorbs at the interface when the particle satisfies $-r^{-1}<\Sigma_{1}<r^{-1}$. The range of the interfacial tensions for the adsorbing state narrows with the increase of $r$.

(c) Energy of the Interface Adsorbing the Parallelepiped Particle with the Microstructured Surface. We calculate the interfacial energy of the liquid-liquid interface adsorbing the parallelepiped surface-active particle as illustrated in Figure 1c. Several situations of adsorption seem possible due to its strong anisotropic shape, and the adsorption energy is important to understand the stability of each condition. Here, we discuss three adsorbing states, that is, (i) state 1 , the main axis and the plane surface of the particle are parallel to the interface; (ii) state 2, the main axis of the particle is perpendicular to the interface; and (iii) state 3 , the main axis of the particle is parallel to the interface and the plane surface of the particle is $\pi / 4 \mathrm{rad}$ to the liquid-liquid interface. In the model, $F^{1}, F^{2}$, and $F^{3}$ are the adsorption energies per particle for states 1,2 , and 3 , respectively. The quantity $a$ is the length of one side of the square, and $l$ is the height of the rectangular parallelepiped particle. 

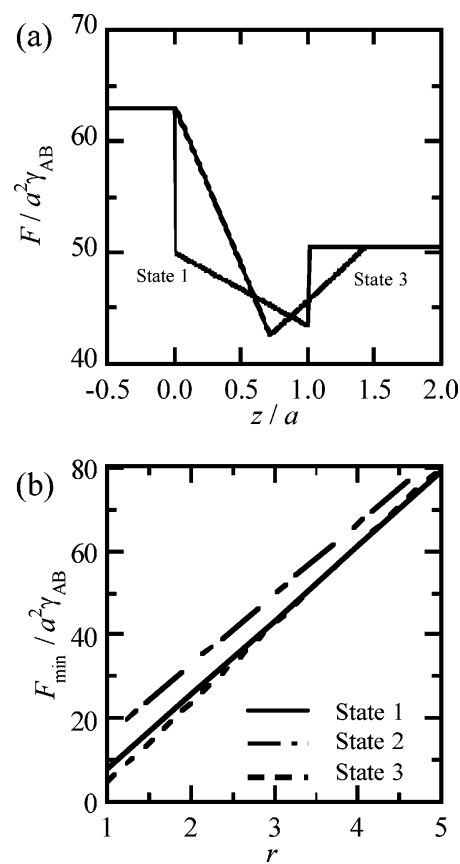

Figure 4. Adsorbing state of the parallelepiped particle. (a) The dimensionless adsorption energy, $F /\left(a^{2} \gamma_{\mathrm{AB}}\right)$, against immersion depth, $z / a$, for the parallelepiped particle at constant length $a$ and $l$ of the particle and the interfacial tensions $\gamma_{i j}$. The parameters are as follows: $\gamma_{\mathrm{AP}} / \gamma_{\mathrm{AB}}=0.4, \gamma_{\mathrm{BP}} / \gamma_{\mathrm{AB}}=0.5, h / R_{\mathrm{d}}=0.1$, and $r=3$. (b) The minimized energy, $F_{\min } /\left(a^{2} \gamma_{\mathrm{AB}}\right)$, against the surface area magnification factor, $r$.

In Figure 4a, we have plotted dimensionless $F /\left(a^{2} \gamma_{\mathrm{AB}}\right)$ when $\gamma_{\mathrm{AP}} / \gamma_{\mathrm{AB}}=0.4, \gamma_{\mathrm{BP}} / \gamma_{\mathrm{AB}}=0.5, l / a=10$, and $r=3$. For state 1 (state 2), the energy changes discontinuously at $z=0$ and $a$ $(l)$, that is, when the plane surface of the particle contacts the liquid-liquid interface. The energy changes linearly with $z$ as a result of the contact of the side surface with liquid $\mathrm{A}$ and attains the minimum at $z=a(l)$. The corresponding minimized energies are given by

$$
\begin{gathered}
F_{\text {min }}^{1}=r\left\{\left(3 a l+2 a^{2}\right) \gamma_{\mathrm{AP}}+a l \gamma_{\mathrm{BP}}\right\}-a l \gamma_{\mathrm{AB}} \\
F_{\text {min }}^{2}=r\left\{\left(4 a l+a^{2}\right) \gamma_{\mathrm{AP}}+a^{2} \gamma_{\mathrm{BP}}\right\}-a^{2} \gamma_{\mathrm{AB}}
\end{gathered}
$$

On the other hand, the energy profile of $F^{3}$ is different from $F^{1}$ and $F^{2}$. The energy decreases with $z$ gradually and attains the minimum at $z=a / \sqrt{2}$. The corresponding minimized energy is given by

$$
\begin{array}{r}
F_{\min }^{3}=r\left(2 a l+a^{2}\right)\left(\gamma_{\mathrm{AP}}-\gamma_{\mathrm{BP}}\right)-\sqrt{2} a l \gamma_{\mathrm{AB}}+ \\
2 r a(2 l+a) \gamma_{\mathrm{BP}}
\end{array}
$$

In Figure $4 b$, we plot $F^{1}{ }_{\min } /\left(a^{2} \gamma_{\mathrm{AB}}\right), F^{2}{ }_{\min } /\left(a^{2} \gamma_{\mathrm{AB}}\right)$, and $F^{3}{ }_{\min } /$ $\left(a^{2} \gamma_{\mathrm{AB}}\right)$ against $r$. They are proportional to $r$, and the slopes for the three states are different from each other. These results indicate that the surface roughness on the parallelepiped particle affects the orientation at the interface at the equilibrium.

(d) Energy of the Liquid-Liquid Interface Adsorbing the Partially Microstructured Particle. Let us consider next the spherical particle partially covered with the microstructured surface, as shown in Figure 1d. We assume here that (i) a part of the particle $\mathrm{P}_{\mathrm{m}}$ is covered by the microstructured surface while the other hemisphere $P_{s}$ is a smooth one and (ii) the surface area of the $\mathrm{P}_{\mathrm{m}}$ region is $r$ times larger than that of the smooth one, as shown in Figure 1d. The interfacial energy, $F$, decreases drastically when the particle in liquid B approaches the interface
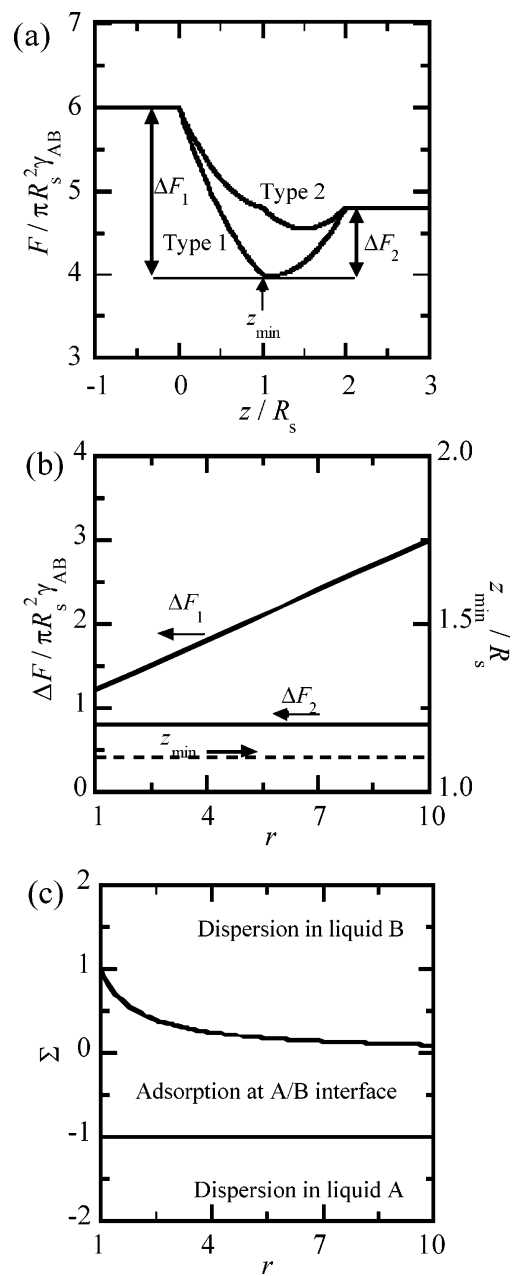

Figure 5. Adsorbing state of the partially microstructured particle. (a) The dimensionless adsorption energy, $F /\left(\pi R_{\mathrm{s}}^{2} \gamma_{\mathrm{AB}}\right)$, against immersion depth, $z / R_{\mathrm{S}}$, at constant radius $R_{\mathrm{S}}$ of the particle and the interfacial tensions $\gamma_{i j}$. The particle approaches from the $\mathrm{P}_{\mathrm{m}}$ side (state 1 ) or from the $\mathrm{P}_{\mathrm{s}}$ side (state 2). The parameters are as follows: $\gamma_{\mathrm{AP}} / \gamma_{\mathrm{AB}}=0.4$, $\gamma_{\mathrm{BP}} / \gamma_{\mathrm{AB}}=0.5, r=5$, and $l=R$. (b) The energy gaps, $\Delta F_{1}$ and $\Delta F_{2}$, and the equilibrium position of the particle, $z_{\min }$, for the particle against the ratio of the particle surface area, $r$. The parameters are as follows: $\gamma_{\mathrm{AP}} / \gamma_{\mathrm{AB}}=0.4, \gamma_{\mathrm{BP}} / \gamma_{\mathrm{AB}}=0.5$, and $l=R$. (c) The state diagram of the particle against the interfacial tensions, $\gamma_{i j}$, and the surface area magnification factor, $r$.

from the $\mathrm{P}_{\mathrm{m}}$ side (Figures 1d and 5a, state 1). In this case, the energy, $F$, decreases with the particle adsorption and the cusp is located at the boundary between the $\mathrm{P}_{\mathrm{m}}$ part and the $\mathrm{P}_{\mathrm{s}}$ part, that is, $z=R$. The energy attains the minimum at $1-r \Sigma(0<$ $\Sigma<1 / r)$ or $1-\Sigma(-1<\Sigma<0)$. The corresponding minimized energy is given by

$$
\begin{aligned}
& F_{\text {min }}=2(r+1) \pi R^{2} \gamma_{\mathrm{BP}}-\pi R^{2} \gamma_{\mathrm{AB}}(1-r \Sigma)^{2} \quad(0<\Sigma<1 / r) \\
& F_{\min }=2(r+1) \pi R^{2} \gamma_{\mathrm{BP}}+ \\
& 2(r-1) \pi R^{2} \gamma_{\mathrm{AB}} \Sigma-\pi R^{2} \gamma_{\mathrm{AB}}(1-\Sigma)^{2} \quad(-1<\Sigma<0)
\end{aligned}
$$

On the other hand, when the particle contacts the interface from the $\mathrm{P}_{\mathrm{s}}$ side, the energy change is smaller than the previous case (Figures 1d and 5a, state 2) The cusp appears also at $z=R_{\mathrm{s}}$. These results indicate that the partial modification of the surface increases the surface activity and fixes the particle direction at the liquid-liquid interface, even if the surface composition of the particle is completely the same. 
When $-1<\Sigma<0$, the energy gaps, $\Delta F_{1}$ and $\Delta F_{2}$, are given by $\Delta F_{1}=-2(r-1) \pi R_{\mathrm{s}}^{2} \gamma_{\mathrm{AB}} \Sigma+\pi R_{\mathrm{s}}^{2} \gamma_{\mathrm{AB}}(1-\Sigma)^{2}$ and $\Delta F_{2}=$ $4 \pi R_{\mathrm{S}}{ }^{2} \gamma_{\mathrm{AB}} \Sigma+\pi R_{\mathrm{S}}{ }^{2} \gamma_{\mathrm{AB}}(1-\Sigma)^{2}$, respectively. In Figure 5b, we plot $\Delta F_{1}, \Delta F_{2}$, and $z_{\min }$ as a function of $r$ when $\gamma_{\mathrm{AP}} / \gamma_{\mathrm{AB}}=0.4$ and $\gamma_{\mathrm{BP}} / \gamma_{\mathrm{AB}}=0.5$. The energy gap $\Delta F_{1}$ is proportional to $r$. However, $z_{\min }$ and $\Delta F_{2}$ do not depend on $r$. In the case of Figure $5 \mathrm{~b}$, the particle is adsorbed at the interface irrespective of $r$, because the particle is adsorbed at the liquid-liquid interface when $\Delta F_{1}, \Delta F_{2}>0$.

The state diagram for the partially microstructured particle is shown in Figure 5c. The particle is adsorbed at the liquidliquid interfaces when $-1<\Sigma<r^{-1}$. The lower value of $\Sigma$ for the adsorbing state does not depend on $r$, because the surface area of the $\mathrm{P}_{\mathrm{s}}$ region is constant irrespective of $r$.

\section{Discussion}

The equilibrium position of the spherical particle is determined not only by the interfacial tension of the particle surface but also by the surface area of the microstructured particle. Figure $2 \mathrm{~b}$ shows that $z_{\min }$ is proportional to $r$. This can be understood by noting that the interfacial energy, $F$, consists of two parts. The first one is the surface energy between the particle $\mathrm{P}$ and the liquids. The second one is the energy change due to the elimination of the interface between liquids $\mathrm{A}$ and $\mathrm{B}$. The effective increment of the particle surface having the microstructured surface influences the first factor directly, although it does not affect the second one. Therefore, the equilibrium position shifts to liquid $\mathrm{A}$ if the particle has a relatively higher affinity to liquid A.

In equilibrium, the contact angle, $\theta_{\mathrm{eq}}$, is related to the interfacial tensions, $\gamma_{i j}$, and the particle surface area by the Wenzel equation, $\cos \theta_{\mathrm{eq}}=-r \Sigma$. This equation shows that the microstructure enhances the wetting properties of the particle. ${ }^{17,18}$ Therefore, the hydrophilic particle having the microstructured surface is more hydrophilic than that having the smooth surface.

How the microstructure affects the adsorbing state of the particle depends on the particle shape. The spherical particle and the disk-shaped particle adsorb at the interface when the particle satisfies $-r^{-1}<\Sigma<r^{-1}$, as shown in Figure 2c and 3c. However, partially microstructured particles are adsorbed at the liquid-liquid interfaces when $-1<\Sigma<r^{-1}$, as shown in Figure 5c. The lower value of $\sum$ for the adsorbing state is contact irrespective of $r$. This required condition is caused by the two-faced geometry of the particle.

The interfacial energy of the interface adsorbing the parallelepiped particle indicates that the microstructure on the particle affects the orientation of the anisotropic particle. When $\gamma_{\mathrm{AP}} /$ $\gamma_{\mathrm{AB}}=0.4, \gamma_{\mathrm{BP}} / \gamma_{\mathrm{AB}}=0.5, l / a=10$, and $r=1$, the particle is adsorbed at the liquid-liquid interface in state 3 , because the energy $F^{3}{ }_{\min }$ is lower than $F^{1}$ and $F^{2}$. This result agrees with that in the previous study by Dong and Johnson. ${ }^{26}$ They investigated the adsorption of prolate and cylindrical particles at a liquid-liquid interface and showed that the planar configuration (states 1 and 3 in the present study) is most stable in both cases. ${ }^{26}$ On the other hand, the particle is adsorbed in state 1 when $r=5$, because $F^{1}$ is lower than $F^{2}$ and $F^{3}$. This shows that the surface roughness is one of the factors which govern the orientation of the particle at the liquid-liquid interface.

In general, one might consider not only the wettability and the surface structure but also the line tension acting on the threephase contact line to control the adsorption behavior of the particle. ${ }^{26}$ The line tension arises as a result of the excess free energy associated with a unit length of the three-phase contact line. In practice, a line tension of $10^{-6} \mathrm{~N}$ would have quite a drastic effect when the size of the particle is less than $20 \mathrm{~nm}$. Moreover, the line tension plays a significant role in determining the adsorption of an acicular particle. ${ }^{26}$ The present theoretical study will be extended in the future to take into account the effect of the line tension.

In this paper, we have shown that the surface activity of the solid particle can be controlled by the microstructure on the particle surface. The strength of the adsorption and the direction of the particle at the liquid-liquid interface are governed by the interfacial tensions and the microstructure of the particle surface. Binks et al. showed that naturally occurring spore particles which have microstructure exhibit as a surface-active agent. ${ }^{27}$ On the other hand, we can inscribe the microstructure on the particle surface. For example, one of the present authors (K.T.) has succeeded in preparing microstructured particles by the spray dry method. ${ }^{28}$ The theoretical results in the present study are useful to understand the behavior of the solid particles and design new functional materials. Some predictions in this work have not yet been proven by the experiments. Further experimental and theoretical studies are necessary to understand the behavior of the surface-active particles.

Acknowledgment. The authors would like to thank Drs. T. Onda, N. Sato, S. Shibuichi, Y. Murase, and H. Mayama for their kind and useful discussions.

Supporting Information Available: Derivations of the interfacial energies. This material is available free of charge via the Internet at http://pubs.acs.org.

\section{References and Notes}

(1) Binks, B. P. Curr. Opin. Colloid Interface Sci. 2002, 7, 21.

(2) Kralchevsky, P. A.; Nagayama, K. Particles at Liquid Interfaces and Membranes; Elsevier: Amsterdam, The Netherlands, 2001.

(3) Nonomura, Y.; Sugawara, T.; Kashimoto, A.; Fukuda, K.; Hotta, H.; Tsujii, K. Langmuir 2002, 18, 10163.

(4) Nonomura, Y.; Fukuda, K.; Komura, S.; Tsujii, K. Langmuir 2003 19,10152

(5) Dickinson, E. An Introduction to Food Colloids; Oxford University Press: Oxford, U.K., 1992.

(6) Kubo, S.; Hirano, I.; Yamada, J. IFSCC VIIIth International Congress 1974, A8. 2374.

(7) Velev, O. D.; Furusawa, K.; Nagayama, K. Langmuir 1996, 12,

(8) Dinsmore, A. D.; Hsu, M. F.; Nikolaides, M. D.; Marquez, M.; Bausch, A. R.; Weitz, D. A. Science 2002, 298, 1006.

(9) Noble, P. F.; Cayre, O. J.; Alargova, R. G.; Velev, O. D.; Paunov, V. N. J. Am. Chem. Soc. 2004, 126, 8092.

(10) Levine, S.; Bowen, B. D.; Paetridge, S. K. Colloids Surf. 1989, 38,325 .

(11) Komura, S.; Hirose, Y.; Nonomura, Y. J. Chem. Phys., in press.

(12) Yu, S.; Colfen, H. J. Mater. Chem. 2004, 14, 2124.

(13) Onda, T.; Shibuishi, S.; Satoh, N.; Tsujii, K. Langmuir 1996, 12, 2125 .

(14) Shibuishi, S.; Onda, T.; Satoh, N.; Tsujii, K. J. Phys. Chem. 1996, 100,19512

(15) Tsujii, K.; Yamamoto, T.; Onda, T.; Shibuichi, S. Angew. Chem., Int. Ed. 1997, 36, 1011.

(16) Shibuishi, S.; Yamamoto, T.; Onda, T.; Tsujii, K. J. Colloid Interface Sci. 1998, 208, 287.

(17) de Gennes, P.-G.; Brochard-Wyart, F.; Quere, D. Capillarity and Wetting Phenomena: Drops, Bubbles, Pearls, Waves; Springer-Vlg: New York, 2003; Chapter 9.

(18) Wenzel, R. N. Ind. Eng. Chem. 1949, 28, 988.

(19) Cassie, A. B. D.; Baxter, S. Trans. Faraday Soc. 1944, 40, 546. 
(20) Nonomura, Y.; Komura, S.; Tsujii, K. Langmuir 2005, 21, 9409.

(21) Nonomura, Y.; Komura, S.; Tsujii, K. Langmuir 2004, 20, 11821.

(22) Nonomura, Y.; Komura, S.; Tsujii, K. J. Oleo Sci. 2004, 53, 607.

(23) Casagrande, C.; Fabre, P.; Raphael, E.; Veyssie, M. Europhys. Lett. 1989, $9,251$.

(24) Fujimoto, K.; Nakahama, K.; Shidara, M.; Kawaguchi, H. Langmuir 1999, 15,4630 .
(25) Hugonnot, E.; Carles, A.; Delville, M. H.; Panizza, P.; Delville, J. P. Langmuir 2003, 19, 226 .

(26) Dong, L.; Johnson, D. T. Langmuir 2005, 21, 3838

(27) Binks, B. P.; Clint, J. H.; Mackenzie, G.; Simcock, C.; Whitby, C P. Langmuir 2005, 21, 8161 .

(28) Mayama. H.; Tsujii, K. Presented at the $11^{\text {th }}$ International Conference on Organized Molecular Films, Sapporo, Japan, June 2005; Paper O55. 